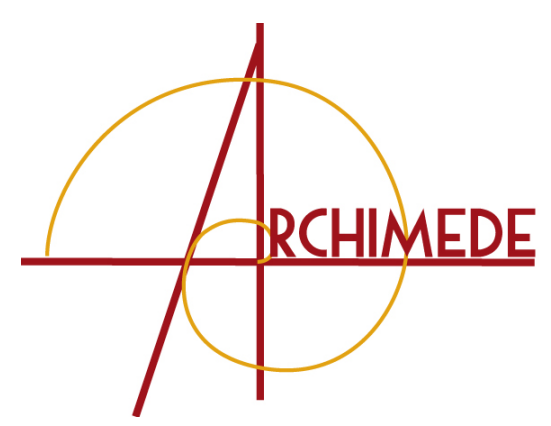

\title{
LE TEMPLE OVALE DE KHAFAJE (MÉSOPOTAMIE CENTRALE) : DE L'ABERRATION ARCHITECTURALE AU RITUEL DE CONSTRUCTION
}

\section{Philippe QUENET}

Université de Strasbourg CNRS-UMR 7044 ARCHIMEDE

pquenet@unistra.fr

Je remercie vivement Anne-Caroline Rendu (université de Genève) de ses stimulantes réflexions et de ses indications bibliographiques. Tout mauvais usage de celles-ci ne serait que de mon fait.

\section{RÉSUMÉ}

Comme les fouilleurs de I'Oriental Institute de Chicago I'ont montré avec brio, les bâtisseurs du Temple ovale de Khafaje (cité du III millénaire av. J.-C. située en Irak central) ont creusé une fosse de $8 \mathrm{~m}$ de profondeur, I'ont remblayée avec du sable et y ont posé les fondations du monument avant d'en monter finalement les murs. La justification de cet énorme travail préparatoire n'est pas fondamentalement d'ordre technique. Elle tient plutôt à des croyances et des pratiques religieuses qui n'ont malheureusement pas laissé de traces explicites dans le sol de Khafaje. Celles-ci sont liées au concept de KI SIKIL,

MotS-CLÉs

architecture, fondations,

rite,

religion, Mésopotamie, période des Dynasties

Archaïques,

Temple ovale,

Khafaje,

KI SIKIL.
As brilliantly demonstrated by the excavators of the Oriental Institute of Chicago, the builders of the Temple Oval in the 3rd millennium B.C. city of Khafadje (Central Iraq) dug an $8 \mathrm{~m}$-deep pit, filled it in with sand and laid upon it the foundation of the monument before they eventually began to construct the walls. The justification for this huge, preparatory work is not technical in essence but rather linked to religious beliefs and practices which, at Khafadje, unfortunately left no self-evident traces in the ground. These are related to the ki sikil concept, the "pure place" in which every temple had to be erected and which may be materialized by the pit of the

KeYWords Temple Oval. architecture, foundation,$$
\begin{array}{r}
\text { rite, } \\
\text { religion, }
\end{array}
$$$$
\text { Mesopotamia, }
$$

Early Dynastic Period, Temple Oval, Khafadje, KI SIKIL. 


\section{INTRODUCTION}

La communication dont cet article dérive avait pour but d'explorer des pistes de réflexion. Il n'en ira pas autrement de cet article lui-même, de sorte qu'il ne faut en aucun cas s'attendre à y voir développée la ligne rigoureuse d'une quelconque démonstration et encore moins à y voir exposés des résultats. Notre point de départ est un simple sujet d'étonnement : les titanesques travaux de terrassement et de fondation qui préludèrent, au milieu du III ${ }^{\mathrm{e}}$ millénaire av. J.-C. [1], à la construction du «Temple ovale » sur le site de Khafaje, dans la vallée de la Diyala en Irak central. Ils ne laissèrent pas, d'ailleurs, d'intriguer en premier lieu les fouilleurs, qui leur cherchèrent un sens. Nous marcherons sur leurs brisées.

Dans la mesure où ces travaux peuvent difficilement s'expliquer dans I'ordre de la matière, leur justification a plus de chance de relever de croyances, et les indices archéologiques recueillis par les fouilleurs de l'Oriental Institute de Chicago (ci-après OIC) pourraient bien, en effet, faire écho à des pratiques en relation avec I'une ou l'autre phase de quelque rituel de construction accompli lors de l'édification de la «maison du dieu», ainsi que les Mésopotamiens appelaient leurs temples. Nous ignorons quasiment tout des rites exécutés durant cette période dans ce genre d'occasion, mais des indices plus ou moins directs, aussi bien textuels qu'archéologiques, permettent au moins d'avérer leur existence.

\section{PRÉSENTATION GÉNÉRALE}

L'établissement antique de Khafaje se situe en rive gauche de la Diyala, à environ $200 \mathrm{~m}$ au sud d'un coude de la rivière. Les vestiges les plus anciens (remontant à la fin du IVe millénaire) ont été identifiés sur la butte $A$, la plus septentrionale et la plus étendue des quatre que les fouilleurs ont distinguées (fig. 1). C'est à cet emplacement que se développa la ville du III e millénaire à l'intérieur d'un mur d'enceinte [2]. Dans sa plus grande extension, elle s'étendit approximativement sur $1 \mathrm{~km}$ de long et $400 \mathrm{~m}$ de large, soit près de 30 ha.

Le Temple ovale se situe en bordure ouest de la moitié sud de la ville, à proximité immédiate du rempart. Il s'insère dans un quartier densément construit, du moins à ce qu'on peut en juger d'après les zones dégagées qui

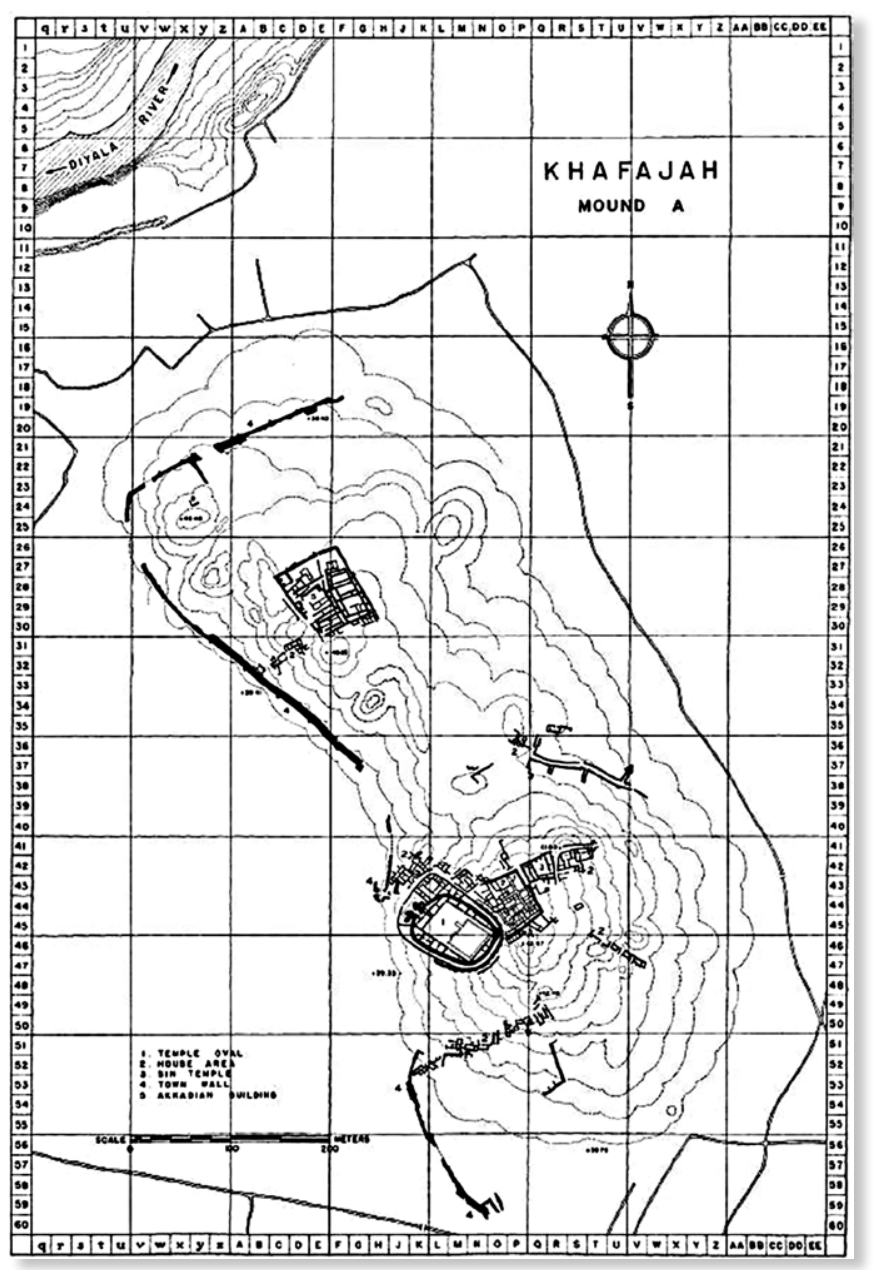

Figure 1 : Plan topographique de la butte A de Khafaje (Delougaz 1940, plate II)

s'étalent du nord-ouest au nord-est du temple. La fouille de l'édifice a commencé en 1930-1931 sous la direction de Conrad Preusser [3] et fut poursuivie au cours des trois campagnes suivantes, jusqu'en 1933-1934, sous la direction de Pinhas Delougaz [4], qui est aussi celui qui assura la rédaction du rapport final [5].

[1] Toutes les dates seront à entendre avant J.-C. dans les pages qui suivent.

[2] V. MARgueron 2013, p. 332-335.

[3] FRANKFORT et al. 1932, p 60-112.

[4] V. FRANKFORT 1933, p. 58-79, FRANKFORT 1934, p. 63-80, et FRANKFORT 1935, p. 32-54, pour les rapports préliminaires.

[5] Delougaz 1940. Sauf indication contraire, toutes les informations concernant le Temple ovale qui apparaîtront dans la suite du texte sont tirées de cet ouvrage. 
La fonction religieuse du bâtiment n'a pas été mise en doute par qui que ce soit, au contraire de certains autres, dans la Diyala ou ailleurs, de dimensions il est vrai plus modestes [6]. La divinité à laquelle le temple était consacré est en revanche inconnue. Une tête de masse d'armes en pierre, d'époque protodynastique et dédiée à la déesse Inanna [7], n'a rien d'un indice décisif en la matière puisqu'elle fut trouvée en subsurface. Deux autres temples protodynastiques du Sud mésopotamien, typologiquement semblables au Temple ovale, ont livré eux aussi du matériel épigraphique. Du premier, celui de Tell el-'Obeid, provient, d'un contexte malheureusement secondaire, une plaquette comportant une dédicace à Ninhursag̃a [8]. Il est avéré en revanche que le second, I'IB.GAL de Tell al-Hiba, ancienne Lagaš, était un lieu de culte voué à Inanna [9].

Trois états de construction principaux ont été établis pour le Temple ovale, numérotés de I à III du plus ancien au plus récent. Trois niveaux d'occupation sont décrits pour l'état I. Les vestiges architecturaux des états II à III étaient néanmoins à ce point érodés et lacuneux qu'il n'a pas été possible aux fouilleurs d'en observer le plan complet. Aussi, pour chacun, deux hypothèses de reconstruction sont-elles avancées. Dans sa reprise de I'analyse des données, Öhnan Tunca conclut que le Temple ovale connut plus certainement quatre états de construction [10].

Grossièrement, ce qui nous est parvenu du Temple ovale fut en usage entre le deuxième et le troisième quart du III $^{\text {e }}$ millénaire. La question est plus débattue en termes de périodisation. L'espace de temps qui couvre les trois premiers quarts du III ${ }^{\mathrm{e}}$ millénaire dans le Sud irakien est divisé en deux grandes périodes, celle du Protodynastique I à III (jusqu'à c. 2350) et celle d'Akkad (jusqu'à c. 2200). Les fouilleurs datèrent la

Figure 2 :

Plan masse du Temple ovale I (Delougaz 1940, plate III)

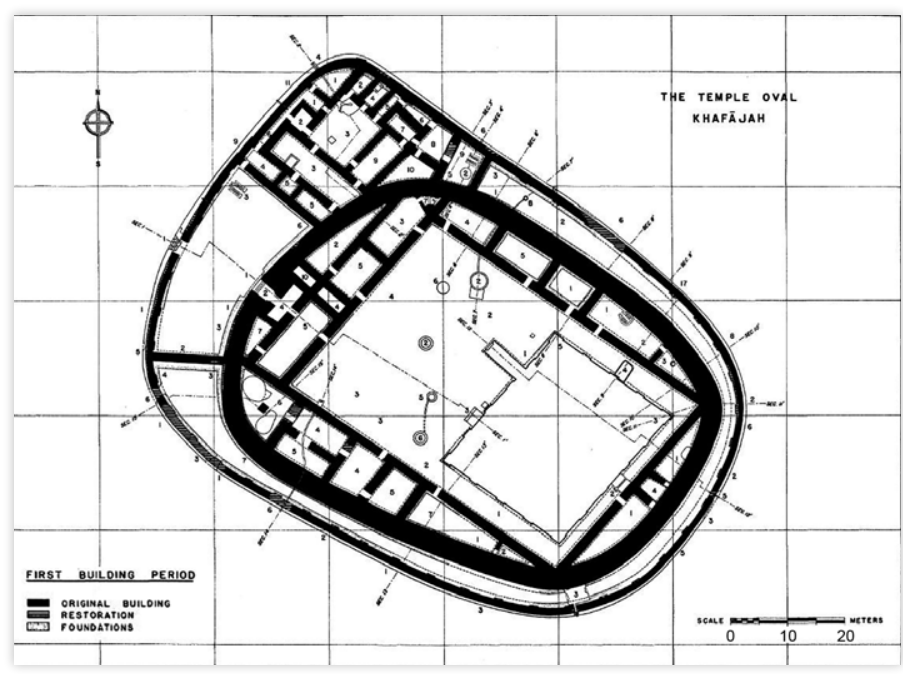

fondation du Temple ovale I du Protodynastique II [11]. Elle pourrait dater plus précisément de la fin de cette période [12] qu'on fait se terminer vers 2600 généralement. L'état III fut initialement rapporté à la fin du Protodynastique III, avec une possible continuité d'utilisation jusqu'au début de la période d'Akkad. Les avis ont ensuite divergé : les uns restent solidaires des fouilleurs [13] tandis que d'autres rejettent le Temple ovale III dans la période d'Akkad commençante [14].

Ces controverses portant sur la stratigraphie ou la chronologie absolue et relative du Temple ovale ne seront pas davantage évoquées car elles n'ont pas une incidence déterminante sur le sujet qui nous occupe. Nous porterons en effet notre attention uniquement sur le mode et les étapes de construction du Temple ovale primitif, celui qui est le mieux connu et dont la mise en chantier et la livraison, peut-être après un an de travaux tout au plus [15], sont à placer approximativement au $x_{x v I I}{ }^{e} s$. Et ce n'est pas tant le bâti en élévation - dont nous ferons une description sommaire - qui nous intéressera que le dispositif de fondation, dont la raison d'être paraît davantage dériver de considérations morales que techniques.

\section{LE BÂTI EN ÉLÉVATION}

La maçonnerie du bâtiment est en briques crues plano-convexes, le matériau de construction typique de la période protodynastique. Le temple doit son nom à la forme de son enveloppe, qui, ni circulaire ni quadrangulaire, a été qualifiée d'ovale à défaut d'une meilleure dénomination (fig. 2). Celle-ci consiste en un mur d'enceinte de 1,5 m d'épaisseur en moyenne et de $300 \mathrm{~m}$ de périmètre. L'édifice est monumental : il mesure une centaine de mètres de long (dans son axe nord-ouest - sud-est) et $74 \mathrm{~m}$ de large au maximum (dans son tiers nord - ouest). Sa largeur s'amenuise en direction du sud-est.

La première enceinte en enclot une seconde, elle aussi ovale, mais plus massive. D'une épaisseur de 3,5 $\mathrm{m}$ en moyenne, elle s'élevait certainement plus haut que la

[6] FOREST 1996 ; FOREST 1999.

[7] Kh. I 636, calcaire, trouvée en M 44:5 au niveau du Temple ovale III (h. $7,7 \mathrm{~cm}$ ).

[8] Hall\&Woolley 1927 ; Delougaz 1938.

[9] CRAWFORD 1972 ; HanSen 1978 ; Hansen 1980-1983.

[10] TUNCA 1984, p. 36-45.

[11] V. à leur suite VÉRTESALJi \& Kolbus 1985 et Evans 2007

[12] PORADA et al. 1992 (écrit en 1986).

[13] Comme PORADA et al. 1992.

[14] GiBSON 1982 en particulier.

[15] Pollock 1999, Table 7.1. 
première (à peu près six mètres contre trois à quatre selon les fouilleurs). Un couloir de 3 à $4 \mathrm{~m}$ sépare les deux murailles, s'élargissant au nord-ouest en une vaste cour. C'est au milieu de celle-ci que débouche l'entrée principale du temple, précédée d'une volée de quatre marches en pierre. En traversant la cour en ligne droite, on arrive devant la porte qui permet de pénétrer, en montant quelques degrés, à l'intérieur de la seconde enceinte. L'espace compris au nord entre les deux enceintes est occupé par un bâtiment organisé autour d'un espace central et nommé « maison $D$ » par les fouilleurs.

L'enceinte intérieure enferme une cour parallélépipédique de $56 \times 38 \mathrm{~m}$ bordée sur tous ses côtés d'une ou deux rangées de pièces. Dans la moitié sud-est de cet espace à ciel ouvert se dressait une plate-forme de plan presque carré $(30 \times 25 \mathrm{~m})$ dont la hauteur conservée, comme pour le reste du bâtiment, n'atteignait pas le demi-mètre. Cependant, elle aurait pu culminer à $c .3 \mathrm{~m}$ à en juger par la longueur de l'escalier qui menait à son sommet $(7,70 \mathrm{~m})$ et par la hauteur de la première marche formée de deux blocs de pierre équarris. Dans leur hypothèse de restitution, les fouilleurs ont choisi de coiffer directement cette plate-forme d'un bâtiment rectangulaire, selon un modèle architectural remontant à l'Obeid (Ve millénaire) [16] et bien attesté à Uruk au IVe millénaire [17].

Le dessin publié par les fouilleurs (fig. 3) a en quelque sorte figé notre vision du Temple ovale I, alors que nombre de points sont discutables. Sans parler du toit en pente de la maison $D$ faisant de l'espace central une espèce d'atrium impluvium, ou du parti pris de faire de cette maison un bâtiment de plain-pied malgré l'épaisseur de ses murs [18], la hauteur restituée des murs d'enceinte du sanctuaire pourrait être sous-évaluée. Les fouilleurs ont opté pour un rapport largeur / hauteur de 1 à 2,5 au maximum; un rapport de 1 à 3 serait

\section{Figure 3}

Restitution en élévation du Temple ovale (d'après Delougaz 1940, frontispice)

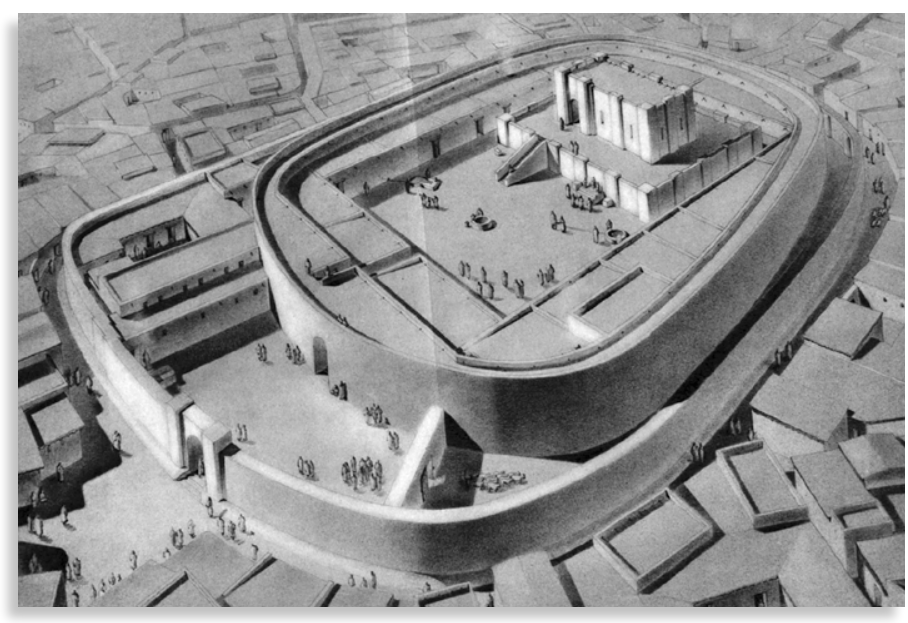

tout aussi plausible. De même la plate-forme auraitelle pu accueillir un deuxième niveau de terrasse [19] et ç'aurait été celui-ci qui aurait formé socle pour un bâtiment sommital [20]. En somme, I'élévation du Temple ovale aurait pu être de plusieurs mètres supérieure à la restitution proposée, ajoutant au caractère imposant de l'édifice.

\section{LES FONDATIONS}

La partie souterraine du Temple ovale n'a rien à envier à sa partie visible : le bâtiment s'enracinait peutêtre aussi profondément qu'il s'élevait en hauteur. Une série de sondages en des points judicieusement choisis permit aux fouilleurs d'établir qu'une gigantesque fosse d'au moins $8 \mathrm{~m}$ de profondeur avait été creusée à l'emplacement même du futur temple. $-8 \mathrm{~m}$ était en effet le niveau de la nappe phréatique à l'époque des fouilles : toute velléité de s'enfoncer plus bas fut donc abandonnée, raison pour laquelle la terre vierge ne fut atteinte nulle part à Khafaje. L'étendue de cette fosse dépassait de peu celle de l'édifice, sauf au nord-est, là où, selon toute vraisemblance, avait été aménagée la rampe permettant de descendre dans l'excavation.

La fosse fut ensuite comblée de «sable pur», pour reprendre la formule de Delougaz. La surface de ce remplissage, fait d'un sédiment rigoureusement naturel, d'origine alluviale ou éolienne et non mêlé à quoi ce soit d'autre, fut damée et nivelée. Les fondations des murs furent posées, selon le principe de la semelle filante. L'espace subsistant entre les fondations fut colmaté avec de I'«argile pure », soigneusement compactée. Cette couche d'assise accueillit ensuite les murs et les sols qui composaient la partie aérienne de l'édifice dont les principales caractéristiques ont été exposées ci-dessus (fig. 4).

Le Temple ovale prit place dans une zone depuis bien longtemps construite, si bien que les terrassiers qui creusèrent la fosse traversèrent des niveaux d'occupation couvrant une période de plus d'un demi-millénaire,

[16] V. en particulier le site d'Abu Shahrain / Eridu (SAFAR et al. 1981).

[17] Le temple Blanc d'Uruk ou bâtiment B, dans le secteur de la ziggurat d'Anu, se présente en effet comme un bâtiment rectangulaire culminant au sommet d'une haute plate-forme (EICHMANN 2007).

[18] On pourrait en dire tout autant des murs des pièces adossées à l'enceinte intérieure.

[19] À Tell Hariri / Mari, les derniers résultats touchant au Massif Rouge soutiennent cette hypothèse (BUTTERLIN 2010, p. 191-192).

[20] L'existence d'un tel bâtiment s'impose au vu des trouvailles faites à Tell el-'Obeid (HALL \& Woolley 1927), d'autant que le bâtiment mis au jour sur ce site, comme nous l'avons dit, est parent du Temple ovale (Delougaz 1938). 


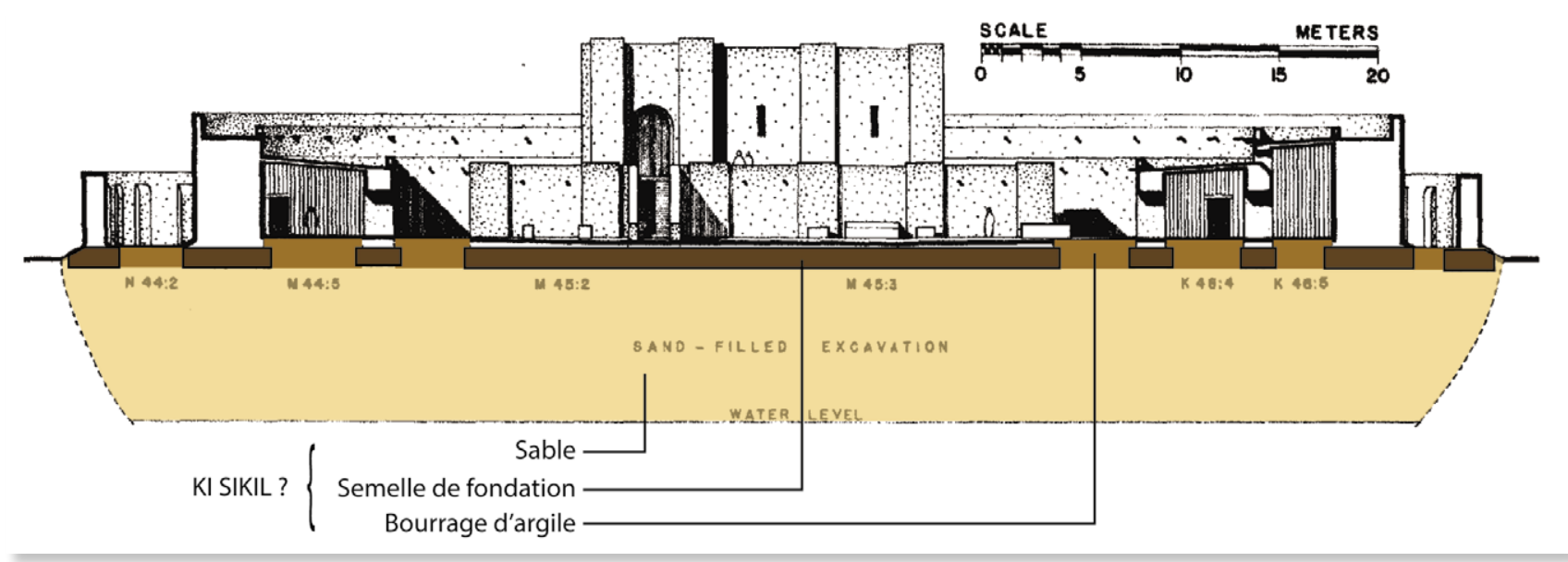

Figure 4 : Section sud-ouest-nord-est du Temple ovale (d'après Delougaz 1940, plate VI)

ceux-là mêmes que les fouilleurs de I'OIC mirent au jour aux abords nord-est du temple en dehors des limites de la fosse [21]. On ignore si, comme eux, les ouvriers antiques rencontrèrent la nappe phréatique et durent renoncer à toucher la terre vierge, mais il y a lourd à parier que tous, malgré les 4500 ans qui les séparaient, furent animés de la même motivation : passer au-dessous du niveau des dépôts anthropiques. Bien que la profondeur réelle de la fosse nous reste inconnue, c'est au moins $64000 \mathrm{~m}^{3}$ de déblais qui furent extraits - dont on se demande d'ailleurs ce qu'ils sont devenus.

Les lois de la statique ne nécessitaient aucunement le creusement d'une telle fosse pour garantir la stabilité des murs de fondations (qui sont du reste enterrées à moins de $2 \mathrm{~m}$ ) et, plus généralement, celle du monument, aussi massif qu'il soit. Par conséquent, des travaux de cette ampleur ne se justifiaient pas, à moins qu'on voulût éliminer tout résidu de présence humaine sous la surface du temple. C'est ce qu'incite à penser la pureté du sable rapporté et des paquets d'argile compressés qui le surmontent. Aussi peut-on conjecturer que le projet d'origine consistait à atteindre la terre vierge pour que la maison du dieu fût bâtie sur un terrain non touché de main d'homme. On aurait donc ici non seulement la manifestation d'une pratique dont on trouve écho dans les textes (comme nous allons le voir), mais aussi, probablement, une trace qui pourrait être liée à un moment particulier au sein de la longue séquence rituelle qui a dû accompagner la construction de l'édifice.

\section{LES RITUELS DE CONSTRUCTION \\ DE TEMPLES AU III" MILLENAIRE DANS LE SUD MÉSOPOTAMIEN}

Les témoignages épigraphiques les plus anciens clairement identifiés à ce jour comme ayant quelque rapport avec des rites de fondation proviennent du Sud mésopotamien et datent du Protodynastique III [22]. Malheureusement, ils ne sont pas de nature à nous renseigner sur les motivations des bâtisseurs du Temple ovale. Les textes de l'époque néo-sumérienne (c. 2100) sont plus loquaces à ce sujet :

\section{1) Inscription sur cylindre du roi Gudéa :}

Cylindre A, Tello, terre cuite, h. $60 \mathrm{~cm}$ (Louvre, MNB 1511), à propos du temple construit pour Ningirsu : [iii 20] The grove(?) (of the House) he let grow on ground pure (= KISIKIL) as the Abzu. [23]

\section{2) Inscriptions sur statues du roi Gudéa trouvées à Tello :}

A) Statue C, «aux épaules étroites », diorite, h. $138 \mathrm{~cm}$ (Louvre, AO 5), à propos du temple construit pour Inanna : [iii 1-5] The clay (for the bricks) he mixed at an absolutely pure site (= KI-UD.UD-GA), and the bricks he formed at a clean place (= KISIKIL). [iii 6-7] He cleansed the foundation pit, made (purifying) fire go over it. [24]

B) Statue E, « aux larges épaules », diorite, h. $140 \mathrm{~cm}$ (Louvre, AO 6), à propos du temple construit pour Bau, et statue $F$, «l'Architecte à la règle », diorite, h. $86 \mathrm{~cm}$ (Louvre, AO 3), à propos du temple construit pour Gatamdu : [iv 1-2] he built (her) house on an absolutely pure site (= KI-UD.UD-GA). [25]

\section{3) Inscription sur brique du roi Ur-Namma provenant d'Ur :}

[21] Delougazet al. 1967, pl. 15.

[22] Cunningham 1997, textes nos 7, 17 \& 18a. Ce dernier est aussi publié dans COOPER 1986, La 1.32.

[23] EdzARD 1997, cylinder A.

[24] EDZARD 1997, statue C.

[25] EDZARD 1997, statues D \& F. 
[1-2] For the god An, king of the gods, his lord, [3-4] Ur-Nammu, king of Ur, [5-6] planted for him a lofty garden (and) built for him a shrine/dais in a pure place (= KI SIKIL). [26]

\section{4) Éloge du roi Ur-Namma :}

City of the finest divine powers, lofty royal throne-dais! Shrine Urim, pre-eminent in Sumer, built in a pure place (= KISIKIL)! [27]

\section{5) Éloge du roi Šulgi}

City worthy of the divine powers, according to its name: shrine Urim, raging storm of Sumer, battleground - and well established! Origin of human seed, consolidating the foundations of the Land, abundance - and well established! Lofty dais of An, pure place (= KISIKIL), holy place, provider of first-fruit offerings for An to refresh himself, dripping with syrup and wine - and well established! [28]

L'idée récurrente est que le sanctuaire a été bâti en un «lieu pur». Cette expression traduit le plus souvent les deux mots sumériens KISIKIL, qui apparaissent aussi inchangés dans un texte littéraire, celui intitulé Enki et I'ordre du monde :

At my command, sheepfolds have been built, cowpens have been fenced off. When I approach heaven, a rain of abundance rains from heaven. When I approach earth, there is a high carp-flood. When I approach the green meadows, at my word stockpiles and stacks are accumulated. I have built my house, a shrine, in a pure place (= KISIKIL), and named it with a good name. I have built my Abzu, a shrine, in ...... [29], and decreed a good fate for it. The shade of my house extends over the ...... pool. [30]

Comme les fouilleurs de I'OIC I'avaient déjà diagnostiqué, la notion de « lieu pur » s'accorde bien avec la situation observée dans les couches profondes du Temple ovale. Le rapprochement satisfait entièrement I'esprit et I'on pourrait en fin de compte s'arrêter là. Ce serait pourtant dommage, car ce serait se cantonner dans l'abstraction, s'en tenir à une vague résonance de vocables ou de concepts désincarnés («lieu pur », « sable pur», « argile pure »). Ce serait surtout se dispenser d'essayer de définir ce que les Sumériens entendaient par «lieu pur » alors que les fondations du Temple ovale peuvent contribuer à nous en forger une idée plus précise.

L'affaire n'est en effet pas une question de traduction, mais de compréhension. Elle ne touche pas au champ lexical, mais au champ sémantique. SIKIL est rendu différemment selon les auteurs. On peut trouver « saint » ou « sacré » par exemple... L'excavation qui a préludé à l'édification du Temple ovale eut manifestement pour but de (re-)créer un KISIKIL, d'aménager, si l'on veut, une « aire sacrée » et il fallait pour cela retourner à la terre vierge, de manière à poser le sanctuaire sur un sol intact et même inviolé, c'est-à-dire que I'Homme n'avait pas foulé. Peutêtre était-ce revenir, par cette sorte de métaphore dont la pensée mythique est coutumière, aux temps d'avant sa création, aux temps primordiaux des seuls dieux, celui des origines. Ainsi le temple aurait-il été ancré de facto dans le numineux.

La mention d'une purification des fossés de fondation par le feu sur une statue de Gudéa est intéressante. Aucune trace de rubéfaction ou de noircissement n'a toutefois été relevée sur les parois de la fosse du Temple ovale. En aurait-on observées s'il avait été possible d'en atteindre le fond ? La formulation employée dans le cylindre A dû au même souverain sous-entend que I'Abzu, le royaume des eaux souterraines, domaine d'Enki / Éa, est d'une pureté suprême. $\mathrm{Si}$, comme les fouilleurs, les ouvriers du III ${ }^{\text {e }}$ millénaire avaient été forcés d'arrêter leur descente au niveau de la nappe phréatique, il semble donc que la sanctification de l'emplacement destiné à accueillir le temple n'aurait pas été mise en péril. La piété n'empêche pas d'avoir l'esprit pratique (ou roublard [31]) : à défaut de terre vierge ou de roche mère, les eaux de subsurface, qui interdisaient de toute façon de creuser plus bas, présentaient elles aussi toutes les qualités requises !

\section{LES RITUELS DE CONSTRUCTION NEO-ASSYRIENS (PREMIERE MOITIÉ DU I' MILLENAIRE)}

Des textes beaucoup plus récents (de la première moitié du Ier millénaire) et issus du Nord-Est irakien nous montrent que l'établissement des fondations d'un temple s'inscrivait dans une chaîne opératoire comprenant deux volets: les phases de travaux, il va de soi, mais également, en étroite corrélation avec celles-ci, un enchaînement complexe de rites [32] dont les différentes étapes ont l'intérêt pour la plupart de pouvoir être mises en regard de données archéologiques et textuelles datant du III millénaire.

[26] FRAYNE 1997, E3/2.1.1.5.

[27] ETCSL, c.2.4.1.3 : Ur-Namma C.

[28] ETCSL, c.2.4.2.15: Šulgi O.

[29] Cette lacune est peut-être à suppléer par la répétition du groupe de mots « in a pure place ».

[30] ETCSL, c.1.1.3.

[31] Il existe en effet une adéquation étonnante - pour tout dire, à l'avantage des Hommes - entre les différentes possibilités offertes aux bâtisseurs (chercher la roche mère ou la terre vierge, la nappe phréatique ou les anciennes fondations) et les principales situations qui pouvaient se rencontrer effectivement sur le terrain.

[32] V. LACKENBACHER 1982 ; LACKENBACHER 1990 ; AMBOS 2004 ; AмBos 2013. 
Des prises de présages précédaient la mise en chantier. Gudéa nous rapporte ainsi comment, dans un rêve, Ning̃irsu, dieu tutélaire de Lagaš, lui inspira le projet de lui (re)bâtir son temple, l'E-ninnu [33]. La fabrication des briques, elle, commençait dans le «mois de la brique » ou, si l'on peut dire, en «briquôse ». Ce mois s'écrivait effectivement au moyen d'un signe $\left(\mathrm{SIG}_{4}\right)$ pouvant se lire « brique crue » ou « Kulla », du nom du dieu connu pour être le maçon d'Enki. Selon les calendriers, d'une région ou d'une époque à l'autre, c'était le deuxième ou troisième mois de l'année, à situer, donc, entre avril et juin pour nous, c'est-à-dire au début de la saison sèche, quand les grosses chaleurs recommençaient à s'abattre sur la campagne mésopotamienne. Le nom de ce mois est attesté à partir du dernier tiers du III $^{\mathrm{e}}$ millénaire (période d'Akkad et d'Ur III).

Deux verbes sont liés à la phase de mise en place des fondations : on les creuse (ušše petû, litt. « ouvrir les fondations »), en prenant soin d'atteindre la roche mère ou la nappe phréatique [34], et on les pose (ušše nadû, litt. « jeter les fondations »). Les observations faites au Temple ovale coïncident rigoureusement avec ces préoccupations et les deux phases de ce processus. Une purification par le feu peut s'en suivre, comme la statue C de Gudéa nous en avait déjà transmis le témoignage. Des offrandes sont faites aux dieux d'En-Bas, dans le royaume desquels on s'est introduit. Dans le cas du Temple ovale, on peut au moins supposer que des précautions de nature exorcistique furent prises quand on traversa les anciennes couches d'occupation, faites de ruines d'habitations mais aussi probablement de sépultures intra-muros : le pouvoir malfaisant d'éventuels fantômes devait être neutralisé [35].

[33] ETCSL, t.2.1.7 (cylindre A). V. aussi SUTER 2000.

[34] On pouvait chercher également à mettre à nu les fondations du temple précédent, mais ce cas de figure ne nous intéresse pas directement ici.

[35] Pour une incantation contre les démons datant du III millénaire (c. 2300), v. KREBERNIK 1984, p. 122-124 ( $n^{\circ} 24$ ), mais il est vrai qu'elle provient du Nord mésopotamien ( $\left.d^{\prime} E b l a\right)$ et que sa vertu est plutôt thérapeutique.

[36] V. ELLIS 1968 et RASHID 1957-1971.

[37] Delougaz 1940 , p. 85-86 \& fig. 78-79. De bien meilleures photos sont accessibles sur DiyArDa.

[38] À propos de ces vestiges en général, v. PARROT 1948, p. 66 $\&$ fig. $25 /$ b. À notre connaissance, il n'existe pas de reproduction récente des deux premiers, qui sont exposés dans une vitrine du musée de I'Orient ancien d'Istanbul. Pour une photographie de AO 2353, voir Atlas.

[39] FRAYNE 2007, E1.9.5.12.

[40] Photographie dans Atlas.

[41] FRAYNe 2007, E1.9.5.4.

[42] Photographie dans Atlas et dessin dans Boese 1971, T 4.

[43] Gudéa est figuré ainsi. V. AO 258, provenant de Tello, dont on trouvera une photographie dans Atlas.
Le phénomène des dépôts de fondation est certainement celui qui offre la meilleure prise à l'archéologie [36]. Ils pouvaient consister en objets anépigraphes divers (parmi lesquels des figurines) ainsi qu'en inscriptions. Les exemples issus de contextes protodynastiques sont nombreux. À Khafaje même, deux lots d'artefacts anépigraphes ont été mis au jour, respectivement aux angles nord et est du second état de la plate-forme (rapporté au Temple ovale II) [37]. Ils reposaient dans la mince couche de sable qui - fait notable - séparait le premier lit de briques de la nouvelle plate-forme et le niveau arasé de la première. Ils se composaient en majorité de matériaux bruts (plaquettes de lapis-lazuli, ardoise, cuivre et autres, fragments de cornaline et cristal de roche, feuilles d'or, fil de cuivre...).

$\mathrm{Si}$, par figurines, on peut entendre ces clous anthropomorphes en cuivre, en général à l'effigie des rois qui ont commandité la construction de tel ou tel bâtiment, alors elles abondent dès le milieu du III ${ }^{\mathrm{e}}$ millénaire. Plusieurs exemples de la seconde moitié du Protodynastique III peuvent être tirés de Tello, comme Ist 1530 à 1531 et AO 2353 [38]. La tête de la figurine est fichée dans un bloc de pierre en forme de brique plano-convexe dont la face plane est couverte d'une inscription mentionnant le nom du roi de Lagaš Enmetena [39]. Les attestations d'inscriptions sur d'autres supports ne manquent pas non plus à partir de cette date. Citons cette plaquette en pierre au nom du même roi Enmetena, longue de $20,5 \mathrm{~cm}$, de provenance incertaine (al-Mada'in ?) et entrée au Louvre en 1971 (AO 24414) [40]. Elle énumère la construction de divers monument religieux consacrés notamment à Ning̃irsu [41].

Seulement ensuite, une pose de la première brique avait lieu. Il est probable qu'une plaque perforée en calcaire au nom du roi Ur-Nanše de Lagaš (h. 39 cm, Tello, Protodynastique III, Louvre, AO 2344) nous en donne une illustration [42]. Elle représente le roi, crâne rasé, nu-torse et vêtu d'une jupe de laine, maintenant de son bras droit un couffin rempli de briques sur sa tête, ce panier en vannerie dont l'iconographie nous montre en maintes autres occasions qu'il servait à transporter divers matériaux de construction. L'inscription associée commémore, certes, la construction d'un temple en I'honneur de Ning̃irsu, d'un second pour la déesse Nanše et d'un troisième monument appelé Abzu-banda, mais elle n'évoque aucunement I'inauguration de I'un ou l'autre de ces chantiers. Des clous de fondation plus tardifs représentent le roi dans une posture similaire [43].

Les parallèles que nous venons de tracer ne visent pas à démontrer que les séquences rituelles attachées à l'établissement des fondations d'un temple étaient identiques au III ${ }^{\mathrm{e}}$ et au I ${ }^{\mathrm{er}}$ millénaire, mais tout au plus à tenter d'ordonner 
et à mettre en perspective des faits archéologiques qui se présentent à nous comme des membra disjecta pour la période protodynastique. En dernière analyse, on retirera de ce rapide examen que le creusement de la fosse du Temple ovale fut vraisemblablement précédé, accompagné et suivi de cérémonies poursuivant des buts divers (divination, hommage, purification, exorcisme...). Beaucoup d'entre elles étaient sans doute de nature à ne pas laisser de traces et, si certaines en laissèrent malgré tout, les archéologues américains n'ont pas eu la chance de les découvrir. Pour exemplaire que fut leur stratégie de fouille, tout ce qu'elle réussit à mettre en évidence est l'existence d'un grand trou.

\section{SIKIL, LE MOT ET LA CHOSE}

Il est troublant que KI-SIKIL désigne également la jeune fille d'âge nubile [44]. Imbus que nous sommes des religions du Livre, nous pourrions être prompts à transposer ce terme en celui de «vierge », malgré toutes les connotations, conscientes ou non, et les préjugés qui s'y rattachent dans notre culture. « Lieu pur », « jeune fille », «vierge », « terre vierge », voilà,entre realia textuels et données matérielles, un réseau de correspondances qui nous arrangerait pour percer le sens de SIKIL... La fosse serait la matrice, le temple viendrait s'y planter, acte qui reproduirait une sorte d'accouplement primordial, dans la veine des interprétations d'Eliade [45].

À l'appui d'une telle hypothèse, on ne peut manquer de citer un texte cosmogonique fameux, connu sous le titre moderne d'Enki et Ninhursag̃a et dont les plus anciennes copies remontent au début du $\mathrm{II}^{\mathrm{e}}$ millénaire :

Les villes sont splendides, ...,

le pays de Dilmun (aussi) était splendide.

Sumer est splendide, ....,

le pays de Dilmun (aussi) était splendide.

Le pays de Dilmun était splendide, le pays de Dilmun était resplendissant,

le pays de Dilmun était vierge (= SIKIL), le pays de Dilmun était immaculé.

Après que l'on a fait s'étendre à Dilmun celui qui était seul,

le lieu où Enki se coucha au côté de son épouse, ce lieu était vierge (= KI... SIKIL...), ce lieu était immaculé.

Après que I'on a fait s'étendre à Dilmun celui qui était seul,

le lieu où Enki se coucha au côté de Ninsikila,

ce lieu était vierge (= KI... SIKIL...), ce lieu était immaculé.

À Dilmun, aucun corbeau ne croassait,

aucun francolin ne cacabait, aucun lion ne se jetait sur sa proie, ni aucun loup n'emportait un agneau.

Inconnu était le chien rabattant les chevreaux, inconnu le cochon mangeur de grains.

Une veuve avait-elle étalé du malt sur le toit, aucun oiseau du ciel ne le picorait, aucune colombe ne se rengorgeait devant elle.

Aucun malade des yeux ne disait: « J'ai mal aux yeux! », ni aucun malade de la tête: « J'ai mal à la tête !».

Aucune vieille femme là-bas ne disait: «Je suis vieille! »,

ni aucun vieillard: « Je suis vieux ! 》.

Aucune jeune fille (= KI-SIKIL) ne se baignait, nulle qui répandît les eaux (usées) dans la ville.

Aucun homme traversant le fleuve ne criait: «... ! ».

Aucun héraut ne faisait sa ronde dans les régions frontières dont il avait la charge.

Aucun aède n'entonnait de chant de joie,

n'entonnait de lamentation aux abords de la ville [46].

Ce n'est qu'ensuite que Dilmun, grâce à l'intervention de plusieurs dieux, devient une terre d'abondance et peuplée de créatures.

Dans ce texte, le KISIKIL paraît naître d'une union, celle d'Enki et de Ninsikila, décrite au début du texte comme l'épouse d'Enki et un plus loin comme sa fille [47]. Ce KISIKIL n'est pas premier. Il est précédé par une sorte de néant, d'incréé, d'où vie et mort, plaisirs et malheurs sont bannis [48]. Passons ici, car ce n'est pas le lieu, sur le jeu verbal à finalité étiologique qui établit l'équation $($ En) $\mathrm{ki}+(\mathrm{Nin})$ sikil(a) = KISIKIL et sur la possible transgression incestueuse à l'origine même de ce «lieu virginal ». Avec, en tête, le schéma chronologique précédemment établi (néant, mise en présence des principes masculin et féminin, émergence de l'étant), retournons plutôt à Khafaje. La fosse ou son creusement pourrait symboliser le (retour au) néant originel, tandis que l'opération par laquelle on la remplit (remblaiement, pose des fondations, montage des murs et rites associés) produirait, elle et elle seule, un KISIKIL (fig. 4) - en fertilisant en quelque sorte l'endroit, en y créant les conditions favorables à l'éclosion de la Vie.

[44] COOPER 2002 ; ePSD, s.v. kisikil. En sumérien, KI SIKIL (« lieu pur ) et KI-SIKIL ( «jeune fille ») partagent en réalité une même graphie. Ce ne sont que les conventions de translitération des assyriologues qui en différencient l'écriture.

[45] ELIADE 1986.

[46] Traduction de Pascal Attinger (http://www.iaw.unibe.ch/ unibe/philhist/ifaw/content/e246526/e255000/e274658/e274665/ e379923/e379932/1_1_1.pdf).

[47] L. 31 du texte : « Ninsikila adressa la parole à son père Enki ». Sur ce lien de filiation, les interprétations sont hésitantes. ATTINGER 1984 , p. 36, adhère au sens propre.

[48] V. la référence en note 47, ainsi que BotTero \& KRAMER 1989, p. 151-164, et Lion 2003 pour la bibliographie. 
Un doute demeure néanmoins. Enki vient s'étendre auprès de Ninsikila. Celle-ci pourrait donc avoir été là avant lui. Il n'y aurait donc pas une opposition si radicale entre incréé / néant et jeune fille (KI-SIKIL) / virginité - peut-être même, à l'inverse, une stricte équivalence. Comment dès lors ordonner selon l'axe du temps l'apparition du «lieu vierge », à la fois préexistant et à venir ? Dans l'implacable royaume de la pensée logique, il y a là des contradictions qui offusquent le bon sens ou qui jettent, comme le dirait Milton Erickson, dans un état de confusion mentale [49], parce que les rapports de lieu et de temps vont à l'encontre des lois ordinaires de la physique. Or s'immerger dans la dialectique onirique de la mythologie mésopotamienne demanderait sans aucun doute une étude beaucoup plus poussée que celle que nous venons de proposer.

\section{BILAN}

D'une superficie au sol de quelque $8000 \mathrm{~m}^{2}$, s'élevant probablement à une hauteur maximale d'au moins 10 à $15 \mathrm{~m}$ et posé sur un remblai de sédiments rapportés de $8 \mathrm{~m}$ de puissance, le Temple ovale fut à sa manière un colosse d'argile bâti sur du sable. L'implantation du temple dans un quartier jusque-là habité impliqua un réaménagement complet de l'espace urbain dans ce secteur [50], mais obligea surtout les bâtisseurs à prendre des mesures drastiques pour respecter des prescriptions religieuses qui voulaient que la « maison d'un dieu » fût édifiée en un KI SIKIL, « lieu pur » ou « terrain vierge », d'où vient qu'on prit la décision de faire place nette en creusant cette fosse béante pour rejoindre une surface terreuse ou minérale, exempte de toute atteinte humaine (voire biologique en général) ou bien jugée telle. Elle fut ensuite comblée d'un matériau ayant les mêmes qualités avant qu'on entamât la construction à proprement parler. Un certain nombre d'indices, quoique indirects pour la plupart, porte à croire que des rites spécifiques, dont la lettre et l'esprit restent presque entièrement à découvrir, accompagnèrent chaque phase des travaux.
Ces spéculations ont, espérons-nous, quelque cohérence, mais elles se hissent à peine au rang d'hypothèses. Pour cela, il faudrait au moins pouvoir appréhender dans toutes ses dimensions, physiques et mentales, ce que recouvre la réalité qui réside selon nous au cœur du sujet, à savoir celle du KI SIKIL. Une première question se pose : est-ce seulement un lieu existant (anciennes fondations, roche mère, nappe phréatique) auquel on peut se contenter de retourner en creusant ? Ou est-ce (aussi parfois ?) un lieu que l'on doit recréer en l'aménageant et en recourant éventuellement à des rites appropriés ? Selon la réponse, ce serait donc le fond de la fosse ou bien son comblement qui constituerait le $\mathrm{KI}$ SIKIL du Temple ovale - encore que le champ des possibles ne soit pas réduit à cette alternative.

La seconde question concerne le sens et les connotations du terme SIKIL dans le contexte qui nous occupe. La confrontation des traductions habituelles («pur», « vierge », « saint ») et du mythe d'Enki et Ninhursag̃a fait apparaître certaines contradictions. Certes, I'exploitation de ce texte, d'interprétation difficile et, au surplus, bien plus tardif que la date de construction du Temple ovale, pose d'importants problèmes méthodologiques. L'on y voit pas moins le kI SIKIL naître d'un acte sexuel. Soit le texte est parodique [51] et associe volontairement des contraires pour créer un effet comique, soit SIKIL n'est pas sans rapport avec la perpétuation de la vie et la fécondité - I'ubérosité comme aurait dit Bottéro - , autant d'aspects que le paradigme de « vierge » est en tout état de cause le moins apte à véhiculer dans notre vocabulaire.

[49] En hypnothérapie, la technique de la confusion mentale consiste à méduser l'entendement pour faire passer un message positif d'acceptation à l'inconscient (v. par ex. ERICKSON 1952). Ici, l'entrée en matière, grandiloquente, et la mise en place rapide d'une intrigue narrative captent immédiatement l'attention, tout en masquant habilement l'incohérence initiale du récit. Le lecteur ou l'auditeur, invité dans le secret des dieux et donc flatté et satisfait, serait en fait feinté, puisque la naissance du KI SIKIL garderait au final tout son mystère.

[50] Vallet 2001 ; Margueron 2012.

[51] LEICK 1994, p. 30-41. 
Atlas : base en ligne des œuvres exposées au musée du Louvre (http://cartelfr.louvre.fr/cartelfr/visite?srv=crt_frm_rs\&langue=fr\&initCritere=false).

DiyArDa : Diyala Archaeological Database (http://networkedblogs.com/Cliby).

ETCSL : The Electronic Text Corpus of Sumerian Literature (http://etcsl.orinst.ox.ac.uk/).

ePSD : The Pennsy/vania Sumerian Dictionary (http://psd. museum.upenn.edu/epsd1/index.html).

Ambos, C., 2004, Mesopotamische Baurituale aus dem 1. Jahrtausend v. Chr., Dresden.

Amвos, C., 2013, «Rituale beim Abriß und Wiederaufbau eines Tempel », dansK. Kaniuth, A. Löhnert, J. L. Miller, A. Otto \&S. Roaf(éd.), Tempel im Alten Orient. 7. Internationales Colloquium der Deutschen Orient-Gesellschaft (11.-13, Oktober 2009, München)(Colloquien der Deutschen Orient-Gesellschaft 7), Wiesbaden, p. 19-31.

Attinger, P., «Enki et Ninhursag̃a », Zeitschift für Assyriologie 74, 1984,p. 1-52.

BoesE, J., 1971, Altmesopotamische Weihplatten, Berlin - New York.

Bottéro, J., \& Kramer, S. N., 1989, Lorsque les dieux faisaient I'homme. Mythologie mésopotamienne, Paris.

Butterlin, P., 2010, «Cinq campagnes à Mari : nouvelles perspectives sur I'histoire de la métropole du moyen Euphrate », Comptes rendus de l'Académie des Inscriptions et Belles-Lettresfasc. 1, p. 171-210.

Cooper, J. S., 1986, Sumerian and Akkadian Inscriptions, I: Presargonic Inscriptions(American Oriental Society, Translation Series 1), New Haven.

Cooper, J. S., 2002, « Virginity in Ancient Mesopotamia », dans S. Parpola \& R. M. Whiting (éd.), Sex and Gender in the ancient Near East: Proceedings of the 4 $7^{\text {th }}$ Rencontre Assyriologique Internationale, Helsinki, July 2-6, 2001, 2 volumes, Helsinki, p. $91-112$.

Crawford, V. E., 1972, «Excavations in the Swamps of Sumer », Expedition 14, p. 12-20.

Cunningham, G., 1997, "Deliver me from Evil". Mesopotamian Incantations 2500-1500 BC(Studia Pohl: Series Maior 17), Roma.

Delougaz, P., 1938, «A Short Investigation of the Temple at Al-'Ubaid », Iraq 5, p. 1-11.

Delougaz, P., 1940, The Temple Oval at Khafajeh (Oriental Institute Publications 53), Chicago.

Delougaz, P., Hill, C., \& Lloyd, S., 1967, Private Houses and Graves in the Diyala Region (Oriental Institute Publications 88), Chicago.

EdzARD, D. O., 1997, Gudea and His Dynasty, The Royal Inscriptions of Mesopotamia: Early Periods 3/1, Toronto.

EichmanN, R., 2007, Uruk. Architektur 1. Von den Anfängen bis zur frühdynastische Zeit (Ausgrabungen in Uruk-Warka Endberichte 14), Mainz am Rhein.

ElIADE, M., 1986, Histoire des croyances et des idées religieuses. I. - De l'âge de la pierre aux mystères d'Eleusis (Bibliothèque Historique Payot), Paris.

ElLIS, R. S., 1968, Foundation Deposits in Ancient Mesopotamia, Yale.

ERICKson, M. H., 1952, «Deep Hypnosis and Its Induction», dans L. M. LeCron (éd.), Experimental Hypnosis, New York, p. $70-112$.

Evans, J. M., 2007, «The Square Temple at Tell Asmar and the Construction of Early Dynastic Mesopotamia, ca. 2900-2350 B.C.E. », The American Journal of Archaeology 111, 4, p. 599-632.

ForeSt, J.-D., 1996, «Les pseudo-temples de la Diyala, ou le contrôle de la population urbaine au Dynastique Archaïque », dans H. Gasche \&B. Hrouda (éd.), Collecteana Orientalia: Histoire, art de l'espace et industrie de la terre. Études offertes en hommage à Agnès Spycket (Civilisations du Proche-Orient. Série I. Archéologie et Environnement 3), Neuchâtel - Paris, p. 97-111.

Forest, J.-D., 1999, Les premiers temples de Mésopotamie (4e et $3^{e}$ millénaires)(British Archaeological Reports. International Series 765), Oxford.

Frankfort, H., 1933, Tell Asmar, Khafaje and Khorsabad. Second Preliminary Report of the Iraq Expedition (Oriental Institute Communications 16), Chicago.

Frankfort, H., 1934, Iraq Excavations of the Oriental Institute, 1932/33. 3rd Preliminary Report of the Iraq Expedition (Oriental Institute Communications 17), Chicago.

Frankfort, H., 1935, Oriental Institute Discoveries in Iraq 1933/34 (Oriental Institute Communications 19), Chicago.

Frankfort, H., Jacobsen, T.,\& Preusser, C., 1932, Tell Asmar and Khafadje. The 1st Season's Work in Ešnunna, 1930/31 (Oriental Institute Communications 13), Chicago.

Frayne, D. R., 1997, Ur III Period (2112-2004 BC) (The Royal Inscriptions of Mesopotamia: Early Periods 3/2), Toronto.

FRAYNE, D. R., 2007, Pre-Sargonic Period (2700-2350 BC) (The Royal Inscriptions of Mesopotamia: Early Periods 1), Toronto.

Gibson, M., 1982, «A Re-evaluation of the Akkad Period in Diyala Region on the Basis of Recent Excavations at Nippur and in the Hamrin », The American Journal of Archaeology 86, 4, p. 531-538. 
Hall, H. R., \& Woolley, C. L., 1927, Al-'Ubaid (Ur Excavations 1), London.

Hansen, D. P., 1978, « Al-Hiba (1968-1976): A Summary of Four Seasons of Excavations », Sumer 34, p. 72-85.

Hansen, D. P., 1980-1983, « Lagash. B. Archäologish », Reallexikon der Assyriologie 6, p. 422-430.

KREBeRNIK, M., 1984, Die Beschwörungen aus Fara und Ebla. Untersuchungen zur ältesten keilschriftlichen Beschwörungsliteratur (Texte und Studien zur Orientalistik 2), Hildesheim - New York.

LACKenbacher, S., 1982, le Roi bâtisseur : les récits de construction assyriens des origines à Teglatphalasar III (Études Assyriologiques cahier $\mathrm{n}^{\circ} 11$ ), Paris.

LACKenbaCher, S., 1990, le Palais sans rival : le récit de construction en Assyrie (Texte à l'Appui, Histoire Classique), Paris.

LeICK G. 1994, Sex and Eroticism in Mesopotamian Literature, New York.

LIoN, B., 2003, «Âges d'or et paradis perdus dans la littérature sumérienne », dans V. Pirenne-Delforge \&Ö. Tunca (éd.), Représentation du temps dans les religions. Actes du colloque organisé par le Centre d'Histoire des Religions de I'Université de Liège (Bibliothèque de la Faculté de Philosophie et Lettres de I'Université de Liège Fasc. 286), p. 55-73.

Margueron, J.-C., 2012, « Notes d'archéologie et d'architecture orientales. 16 - De la strate à la "couche architecturale": réexamen de la stratigraphie de Tuttub/Khafajé (I - L'architecture civile) », Syria 89, p. 59-84.

Margueron, J.-C., 2013, Cités invisibles. La naissance de l'urbanisme au Proche-Orient. Approche archéologique, Paris.

Parrot, A., 1948, Tello (1877-1933), Paris.

Pollock, S., 1999, Ancient Mesopotamia. The Eden that Never Was, Cambridge.

Porada, E., Hansen, D. P., Dunham, S., \& Babcock, S. H., 1992, « The Chronology of Mesopotamia, ca. 7000-1600 B.C. 》, dans R.-W. Ehrich (éd.), Chronologies in Old World Archaeology (3rdedition), 2 volumes, Chicago - London, p. 77-121 (vol. I) \& 90-124 (vol. II).

RASHID, S. A., 1957-1971, « Gründungsbeigaben 》, Reallexikon der Assyriologie 3, p. 655-661.

Safar, F., Mustafa, M. A., \& Lloyd, S., 1981, Eridu, Baghdad.

Suter, C. E., 2000, Gudea's Temple Building. The Representation of an Early Mesopotamian Ruler in Text and Image (Cuneiform Monographs 17), Groningen.

TunCA, Ö., 1984, I'Architecture religieuse protodynastique en Mésopotamie, 2 volumes (Akkadica Supplementum II), Leuven.

VALLeT, R., 2001, « Khafadjé ou les métamorphoses d'un quartier urbain au III millénaire », dans C. Breniquet \&C. Képinski (éd.), Études mésopotamiennes. Recueil de textes offerts à Jean-Louis Huot, Paris, p. 449-461.

Vértesalui, P. P., \& Kolbus, S., 1985, « Review of Protodynastic Development in Babylonia », Mesopotamia 20, p. 53-109. 